\title{
Médiévales
}

Langues, Textes, Histoire

68 | printemps 2015

Langues d'Angleterre

\section{Patrick GAUTIER-DALCHÉ (dir.), La Terre. Connaissance, représentations, mesure au Moyen Âge}

Turnhout, Brepols («L'Atelier du médiéviste », n 13), 2013, 710 p.

\section{Hélène Noizet}

\section{(2) OpenEdition}

Journals

Édition électronique

URL : https://journals.openedition.org/medievales/7521

DOI : 10.4000/medievales.7521

ISSN : 1777-5892

Éditeur

Presses universitaires de Vincennes

Édition imprimée

Date de publication : 15 juin 2015

Pagination : 197-200

ISBN : 978-2-84292-430-0

ISSN : 0751-2708

Référence électronique

Hélène Noizet, «Patrick gautier-dalché (dir.), La Terre. Connaissance, représentations, mesure au Moyen Âge », Médiévales [En ligne], 68 I printemps 2015, mis en ligne le 09 juillet 2015, consulté le 22 avril 2022. URL : http://journals.openedition.org/medievales/7521; DOI : https://doi.org/10.4000/ medievales.7521

Ce document a été généré automatiquement le 22 avril 2022.

Tous droits réservés 


\title{
Patrick GAUTIER-DALCHÉ (dir.), La
}

\section{Terre. Connaissance, représentations,}

\section{mesure au Moyen Âge}

Turnhout, Brepols («L'Atelier du médiéviste », n 13), 2013, 710 p.

\author{
Hélène Noizet
}

\section{RÉFÉRENCE}

Patrick GAUTIER-DALCHÉ (dir.), La Terre. Connaissance, représentations, mesure au Moyen Âge, Turnhout, Brepols (« L'Atelier du médiéviste », n 13), 2013, 710 p.

1 À l'instar des précédents volumes de la collection « l'Atelier du Médiéviste », cet opus le $n^{\circ} 13$ - est un manuel qui présente, édite et traduit des documents médiévaux, en l'occurrence de type géographique, de la fin de l'Empire romain à la Renaissance. L'ouvrage comporte deux parties. Intitulée « Une image du monde. La géographie dans l'Occident médiéval ( $\mathrm{v}^{\mathrm{e}}-\mathrm{XV}$ siècle) », la première partie, rédigée par Christiane Deluze, est une présentation générale de la production documentaire géographique médiévale. Elle est composée de quatre chapitres chronologiques, correspondant au temps des auctoritates ( $\mathrm{V}^{\mathrm{e}}-\mathrm{XI}^{\mathrm{e}}$ siècle), des questionnements ( $\mathrm{XI}^{\mathrm{e}}$-début $\mathrm{XIII}^{\mathrm{e}}$ siècle), des voyageurs (milieu du XIII ${ }^{\mathrm{e}}$-début $\mathrm{du} \mathrm{XV}^{\mathrm{e}}$ siècle) et des humanistes ( $\mathrm{Xv}^{\mathrm{e}}$ siècle). Elle propose un tour d'horizon assez large de différents types de documents, tout en étant pourvue de notices bibliographiques substantielles. La seconde partie, qui constitue le corpus documentaire proprement dit, se compose de six chapitres organisés thématiquement, avec un resserrement progressif de l'échelle. Chacun de ces chapitres est introduit par une présentation plus ou moins synthétique, d'une longueur variable; suivent les documents sélectionnés pour le thème en question (au total 110), images ou textes édités dans la version latine ou vernaculaire, traduits et commentés avec des connaissances techniques utiles à leur compréhension. Pour qui veut travailler sur ces 
documents, cet ouvrage est un excellent instrument de travail, très bien fait et très érudit. Il constitue une porte d'entrée qui n'avait pas d'équivalent jusque-là.

2 La première partie vise à restituer les finalités et le sens social de ces productions documentaires indépendamment d'une perspective téléologique et progressiste: il s'agit, lors de l'analyse d'un document particulier, de ne pas considérer la redécouverte de Ptolémée au Xve siècle comme le point d'arrivée vers lequel tendrait inéluctablement toute production antérieure, comme si elle en était le but ultime. Les auteurs militent donc pour réinsérer l'interprétation de ces documents dans le contexte du savoir de chaque époque, sans les discréditer au motif qu'ils ne sont pas géographiquement vrais d'un point de vue contemporain. De même, les textes classiquement repérés comme des points saillants du progrès géographique sont contextualisés et remis à leur juste place. Ainsi est en-t-il, par exemple, du célèbre passage de Guillaume de Rubrouck sur la fermeture de la mer Caspienne (p. 72), de l'approfondissement des questions théoriques à partir d'Albert le Grand et Roger Bacon (p.117), de l'ascension du mont Ventoux (p. 128), qui n'est évidemment pas l'apparition de la notion moderne du «moi», mais une méditation augustinienne sur la vanité des choses... Dans cette approche d'histoire culturelle, il s'agit de comprendre les interrogations que n'ont cessé d'avoir les clercs médiévaux, en relation avec la compréhension du texte biblique: «L'Océan est-il vraiment infranchissable, dans quelle proportion la terre est-elle recouverte par l'eau, les zones torrides et polaires sont-elles vraiment inhabitables, des humains vivent-ils de l'autre côté de la terre?» (p.156) Cette question des antipodes les taraude en raison de l'unicité de l'origine humaine d'après la Genèse: dans quelle mesure des peuples séparés par des obstacles géographiques aussi radicaux que l'océan, les déserts torrides ou les pôles glacés peuvent-ils tous descendre d'Adam? Autre ferment important de la réflexion géographique des médiévaux, l'astrologie: il leur était nécessaire de situer assez précisément un lieu sur la terre afin de mesurer les influences astrales auxquels il était soumis. Ces diverses finalités furent bien à l'origine d'un élargissement du savoir géographique au cours du Moyen Âge, dont ce livre témoigne à merveille. Thème récurrent de l'ouvrage, la chaîne de la transmission documentaire est évoquée notamment pour l'œuvre de Ptolémée, depuis l'Antiquité tardive jusqu'à l'Ymago mundi de Pierre d'Ailly (rédigée vers 1410), dont Christophe Colomb possédait un exemplaire sur lequel il avait annoté le célèbre passage sur la petitesse de la mer entre l'Espagne et l'Inde. Or ce n'est pas l'Ymago mundi qui reprenait le mieux les apports de la Géographie de Ptolémée, mais une autre des œuvres de Pierre d'Ailly (le Compendium cosmographie). Cela montre à la fois que la transmission du savoir n'est pas linéaire, et qu'il y a des erreurs productrices de savoirs. La redécouverte de Ptolémée $a u x^{e}$ siècle donne lieu à un bouillonnement de la production cartographique et du savoir. Pour autant, Ptolémée n'est pas repris servilement : il est à la fois source d'inspiration et mis à jour, confronté aux expériences oculaires des navigateurs. L'élargissement du monde résulte bien d'innovations culturelles médiévales. Le Moyen Âge a ainsi été plus inventif que ce que l'on en dit habituellement: cette publication montre bien cette extension progressive du savoir géographique, par petites touches, et à partir des propres questions que se posaient les clercs médiévaux. En témoigne ainsi l'enrichissement du vocabulaire géographique, signalé entre $\mathrm{xI}^{\mathrm{e}}$ et $\mathrm{xIII}$ siècle sous la forme d'un simple encart (p. 120) que l'on aurait aimé voir un peu plus développé. Par ailleurs, dans cette première partie, on peut parfois regretter que l'auteure ne donne pas son point de vue dans certains débats bien identifiés, comme dans ceux concernant Henri le Navigateur ou la carte de Christophe Colomb (p. 148). 
3 Dans la seconde partie, le premier chapitre, rédigé par Patrick Gautier-Dalché, sur la Terre dans le cosmos comporte de très bonnes pages sur le mythe, construit au XIX siècle, de la Terre plate au Moyen Âge. Les hypothèses avancées pour expliquer que le mythe dure encore aujourd'hui - l'absence de légitimité de la modernité technologique et le relativisme épistémologique actuel - gagneraient toutefois à être précisées.

Dans le chapitre sur l'espace habité, qui réunit quarante-six documents, Nathalie Bouloux rappelle qu'il y eut un renouvellement profond de la géographie au Moyen Âge, notamment par extension des domaines géographiques étudiés, mais dans des cadres de pensée hérités de l'Antiquité, cadres qui n'exploseront qu'à l'époque moderne. Elle revient sur la réception des autorités antiques et les innovations de la transmission médiévale, qui en transforme progressivement le contenu et ne se limite pas à une copie servile des classiques. Il n'y a pas d'opposition simple entre un savoir théorique de l'autorité issu des textes et un savoir empirique issu des voyages et découvertes. À partir des annotations de lecture des manuscrits, elle établit leur réception, à l'instar d'une tendance historique actuelle qualifiée de «tournant documentaire» (voir en particulier les travaux de Pierre Chastang et d'Étienne Anheim).

5 Dans le chapitre sur l'espace maritime, Emmanuelle Vagnon fait une histoire des cartes maritimes dénommées portulans. On regrette évidemment qu'il n'y ait aucune reproduction d'images : même en noir et blanc, il aurait été utile de voir cette fameuse toile d'araignée des lignes des vents si bien décrite par l'auteure. Ces cartes présentent un tracé relativement exact respectant une échelle des distances et les directions de la boussole. Il y a, à cet égard, une remarque très intéressante sur les usages pratiques de ces cartes (p. 453) : elles avaient une bien plus grande utilité en étant scientifiquement fausses mais techniquement congruentes avec les pratiques de la navigation d'alors car, pour les marins qui naviguaient à la boussole, les cartes en accord avec une direction magnétique étaient bien plus utiles que des cartes montrant de façon exacte les coordonnées latitudes/longitudes, qui étaient peu utilisées dans ce contexte au Moyen Âge. Les portulans permettaient ainsi d'avoir une vue d'ensemble des côtes pour retrouver sa route après une tempête.

6 Dans le quatrième chapitre, Christine Gadrat rassemble des récits de voyage de nature très (trop ?) variable, concernant tout aussi bien les pèlerinages, les voyages lointains de type missionnaire, les expéditions commerciales, les conquêtes militaires, etc. L'auteure remarque fort justement que les récits de voyage ne sont jamais la stricte retranscription de la pure observation du voyageur: il part avec son bagage intellectuel, ses lectures et les insère dans son récit. Dans la mesure où un des credo de l'ouvrage est de resituer dans son contexte la production documentaire, on peut se demander toutefois s'il était pertinent de regrouper tous ces récits dans la catégorie, certes commode, de "voyage». Quel point commun, du point de vue de la finalité, entre ces diverses entreprises? Il est indiqué qu'il réside dans le voyage lui-même, qui procure un sentiment de la distance parcourue, d'éloignement et de dépaysement, ce qui concerne toutefois plus la forme du déplacement que son sens social.

7 Le cinquième chapitre, de Paul Fermon, se caractérise par une grande unité de l'objet étudié, à savoir les cartes locales ou à grande échelle. Après une introduction synthétique solide, le grand mérite de ce chapitre est de faire un recensement très détaillé des ressources documentaires en la matière. En parlant du furor geographicus qui saisit les sociétés à partir des $\mathrm{XIV}^{\mathrm{e}}-\mathrm{XV} v^{\mathrm{e}}$ siècles, l'auteur rappelle bien que ce type de 
production cartographique est essentiellement le fait des deux derniers siècles du Moyen Âge. Leur usage juridique est clair à partir du XIv siècle: ces plans sont majoritairement réalisés lors d'actions en justice quand il y a contestation d'un droit de propriété. Le plan remplace alors le «jour de montrée», lorsque les magistrats venaient visiter en personne les lieux objets du litige.

Enfin, le sixième chapitre, d'Armelle Querrien, intitulé «Techniques et pratiques de la mesure du sol », concerne principalement l'arpentage. Il présente « l'acte de mesure de la terre dans ses cadres technique, économique et social », en s'appuyant sur un dossier documentaire qui comprend, comme il se doit, des extraits de l'œuvre de Bertran Boysset, auteur du principal traité d'arpentage médiéval - mais non unique, comme il est indiqué p. 627. Il existe en effet un autre livre sur la mesure des terres, qui est un véritable traité technique de l'arpentage médiéval ${ }^{1}$ : rédigé en Prusse à la fois en latin et en allemand, vers 1393-1407, il a été édité à la fin du xIX siècle par Hans Mendthal sous le titre de Geometria Culmensis. Dans le dossier documentaire de ce chapitre, on trouve également des actes de la pratique et des documents normatifs qui évoquent les questions de mesure, partage, bornage et délimitation de terres, notamment en cas de désaccord. Par ailleurs, s'il n'y a pas de filiation directe entre les traités des agrimensores romains et l'arpentage médiéval, l'auteure rappelle à bon droit qu'il a toujours existé une pratique bien vivante de l'arpentage.

Pour finir, on peut formuler quelques critiques très circonscrites. Une première s'adresse à l'éditeur : ici la reproduction en couleur des documents n'aurait pas été du luxe. Sur le fond, on peut regretter un point de vue un peu mono-disciplinaire et l'absence de références bibliographiques liées au sujet, par exemple à Jean-Marc Besse, dont les ouvrages font référence sur le savoir géographique à l'époque humaniste, certes au XVI ${ }^{\mathrm{e}}$ siècle, mais qui aurait pu être mentionné, ou à Gérard Chouquer (La Terre dans le monde romain: la proximité du titre est tout de même éloquente; ou encore Arpenteurs et géomètres lorsqu'est évoqué l'arpentage antique), Philippe Cardinali (L'Invention de la ville, qu'on aurait attendu dans le passage sur la place de la ville comme objet pictural, p. 592), et Christian Grataloup (L'Invention des continents). Cette mise à jour des connaissances sur le savoir géographique médiéval reste très interne à la discipline historique médiévale. Que l'ouvrage soit centré sur l'univers de la médiévistique n'est pas en soi critiquable, d'autant qu'il le fait très bien et que c'est bien l'esprit de la collection. Simplement, nous ne partageons pas le point de vue de Patrick Gautier-Dalché qui récuse le spatial turn des sciences humaines et sociales, qu'on ne peut se contenter d'identifier à Michel Foucault (p.11). Sur cette question auraient pu être convoquées d'autres références qui ne sont pas ou très peu citées: pas un mot sur les réflexions, pour se limiter à l'histoire médiévale, d'Anita Guerreau (citée furtivement p.643) ou de Dominique Iogna-Prat, aucun mot sur des publications de référence en géographie (par exemple, Jacques Lévy, Le Tournant géographique. Penser l'espace pour lire le monde, 1999), dont certaines intègrent pourtant les contributions des historiens et archéologues (cf.Jacques Lévy, Michel Lussault éd., Dictionnaire de la géographie et de l'espace des sociétés, 2003, rééd. 2013), et quasiment rien sur les systèmes d'information géographique qui, au-delà d'un effet de mode, permettent bel et bien de poser de nouvelles questions (très courte mention p.625, mais sans référence bibliographique).

10 Ces regrets n'enlèvent rien aux très grandes qualités, synthétiques et documentaires, qui feront de cet ouvrage, à bon droit et de manière durable, une référence et une porte 
d'entrée pour tout médiéviste cherchant à travailler sur des documents de nature géographique. Cette nouvelle et riche mise à disposition de dossiers documentaires, particulièrement bien traduits et présentés, permettra certainement de mieux faire connaître ce pan, jusque-là trop négligé ou caricaturé, du savoir médiéval.

\section{NOTES}

1. H. MENDTHAL éd., Geometria Culmensis. Ein agronomischer Tractat aus der Zeit des Hochmeisters Conrad von Jungingen (1393-1407), Leipzig, 1886. Voir la présentation qui en est faite dans: G. CHOUQUER, Arpenteurs et Géomètres. Dix portraits de l'Antiquité au XVIII siècle, Paris, 2013.

\section{AUTEURS}

\section{HÉLÈNE NOIZET}

LaMOP, Université Paris $1 /$ CNRS 